\title{
STEADY-STATE THIRD-ORDER NONLINEAR SPECTROSCOPY IN INHOMOGENEOUS MEDIA
}

\author{
(Presented by K. K. Rebane)
}

An analysis and model calculations are presented to clarify how both the electronic and vibrational inhomogeneities with no correlation between them influence the most relevant resonant steady-state third-order nonlinear optical mixing processes (CARS, CSRS, polarization spectroscopy and Raman gain (loss)). It is shown that all these processes may be divided into three groups: 1) processes yielding almost homogeneous spectra: both inhomogeneities are greatly reduced; 2) processes yielding quasihomogeneous spectra: inhomogeneities are present, but the electronic one is not manifest in the spectrum; 3 ) processes yielding completely inhomogeneous spectra. The suppression of inhomogeneous broadening allows to study the excited electronic states and the ultrafast relaxation processes in inhomogeneous media more effectively than in linear spectroscopy.

In recent years, thanks to the advances of tunable lasers, besides the traditional optical spectroscopic methods there has been a remarkable development in the field of nonlinear spectroscopic techniques $\left[{ }^{1}\right]$. Among them the methods governed by the third-order nonlinear polarization $P^{(3)}$ have attracted considerable attention. This is due to the fact that $P^{(3)}$ can occur in any medium, methods are relatively simple, and they yield unique spectroscopic information that cannot be achieved by traditional techniques. It is worth mentioning that coherent excitation enables one to prepare optically the sample under study in a necessary way.

There also exist extensive investigations of ultrafast dynamic properties of matter and light-matter interactions. Most experiments have been made in the time domain with the aid of a sophisticated short-pulse technology $\left[{ }^{1-4}\right]$ or coherent optical transient techniques $\left[{ }^{1,5,6}\right]$. On the other hand, it is well known that in linear spectroscopy any relaxation process results in a spectral broadening. (In impurity systems see e. $g$. $\left.\left[{ }^{7,8}\right]\right)$. This holds, in a sense even to a larger extent, in nonlinear spectroscopy, although dependences there are not so simple and straightforward $\left[{ }^{9-13}\right]$. This means that a lot can be learned, about ultrafast relaxation processes from the steady-state spectra by measuring only the width of spectral components.* This greatly enhances the spectral region of the optical transitions used in the study of ultrafast dynamical properties of matter and is particularly valuable for the investigation of processes faster than the shortest available laser pulses.

In all cases the determination of spectral and dynamic information is directly connected with the problem of whether a specific transition of a material is broadened homogeneously or inhomogeneously. The latter

* About another steady-state frequency domain technique by hot luminescence measurements for the study of ultrafast energy relaxation see. e. g. [14]. 
case is realized very often, which essentially interferes with the study of homogeneous properties of matter. Within the framework of linear spectroscopy this obstacle is difficult to overcome. In condensed phases only an almost trivial method of investigation of highly diluted samples [ $\left.{ }^{15}\right]$ and the method of laser-induced fluorescence-line narrowing can be mentioned $\left[{ }^{16,17}\right]$. Generally this is not the case in nonlinear spectroscopy. Numerous nearly inhomogeneity-free high-resolution steady-state methods have been developed during the last decade that are applicable to gaseous media $\left[{ }^{18}\right]$. In the case of condensed media the situation is less promising though not hopless. Aside from the now well-elaborated hole burning method $\left[{ }^{19,20}\right]$, certain third-order optical mixing processes exist that are almost insensitive to the inhomogeneous broadening $\left[{ }^{9-13,21,22}\right]$. Nevertheless, in some cases the effect of the inhomogeneous broadening on the measured spectra and the relaxation parameters determined is rather obscure.

The aim of the present paper is to analyze and demonstrate, on the basis of model calculations, how both the electronic and vibrational inhomogeneities separately influence the most relevant resonant** steadystate third-order nonlinear optical mixing processes. Although vibrational inhomogeneities are usually $10-100$ times less than corresponding electronic inhomogeneities $[2,15,23,24]$, they can essentially hinder the determination of medium-speed relaxation characteristics from steadystate spectra. This is not taken into account by other investigators.***

Third-order nonlinear polarization may be found by solving the Schrödinger equation in density matrix formalism and by using the perturbation expansion of the density matrix elements. We restrict ourselves to two input laser frequencies $\omega_{1}$ and $\omega_{2}$ and to a matter with no resonant states at $2 \omega_{1}, 2 \omega_{2}$ and $\omega_{1}+\omega_{2}$. The Fourier components of the steady-state solutions that oscillate at various frequencies, correspond to different nonlinear phenomena: sum-frequency generation $\left(3 \omega_{1}, 3 \omega_{2}, 2 \omega_{1}+\omega_{2}\right.$ and $\left.2 \omega_{2}+\omega_{1}\right)$, coherent anti-Stokes Raman scattering (CARS) - and coherent Stokes Raman scattering (CSRS)-type processes $\left(2 \omega_{1}-\omega_{2}\right.$ and $\left.2 \omega_{2}-\omega_{1}\right)$, Raman gain and loss or various kinds of polarization spectroscopy $\left(\omega_{1}\right.$ and $\left.\omega_{2}\right)$, depending respectively on the experimental arrangement.

Taking both the electronic and vibrational inhomogeneities into account, the nonlinear polarization has to be averaged over these distributions and then the macroscopic third-order polarization is as follows:

$$
P^{(3)}(\omega)=N \iint\left(\mu_{13} \stackrel{(3)}{31}^{(3)}+\varrho_{13}^{(3)} \mu_{31}\right) g\left(\omega_{0}\right) g(\Omega) d \omega_{0} d \Omega,
$$

where $\mu_{13}$ is the corresponding electric dipole matrix element which in the Born-Opperheimer approximation is equal to $\mu_{12} F$ ( $F$ is the Frank-Condon overlap factor), $\mathrm{Q}_{13}^{(3)}$ - the third-order density matrix element, $N$ - the density of molecules, $g\left(\omega_{0}\right)$ and $g(\Omega)$ are respectively the electronic and vibrational inhomogeneous distribution functions with corresponding halfwidths at half maximum (HWHM) $\Delta \omega$ and $\Delta \Omega$. For an analysis of a certain process, one must know the exact form of $\varrho_{13}^{(3)}$.

Let us start the analysis with the polarization spectroscopy $\left[{ }^{25}\right]$ method similar to the one discussed in $\left[{ }^{26}\right]$. In this case a polarized pumping wave at $\omega_{2}$ fixed on a one-photon resonance $0-0^{\prime}$ transition of

\footnotetext{
** The processes, where at least one mixed light wave is in resonance with certain electronic transition of the matter, are called resonant here.

**:s In $\left[{ }^{2}\right]$, where the Doppler broadening in a gas medium is considered, a certain correlation between two kinds of inhomogeneities is assumed.
} 
a molecular system with a peak at $\omega_{0}$ creates an intensity and frequencydependent birefringence and dichroism that, in its turn, changes the polarization of a weaker probing wave at $\omega_{1}$. The probing wave is scanned through the vibrational levels $\Omega^{\prime}$ of the excited state and a new polarization component at the frequency $\omega_{1}$ serves as a detected signal. With the choice of $\omega_{1} \approx \omega_{0}+\Omega^{\prime}$, this process in a homogeneous system is described by the third-order off-diagonal density matrix element $\left[{ }^{22,}{ }^{26}\right]$ :

$$
\mathrm{Q}_{13}^{(3)}\left(\omega_{1}\right)=\frac{\left|\mu_{12} E_{2}\right|^{2} \mu_{12} F E_{1} \mathrm{e}^{-i \omega_{1} t}}{(i \hbar)^{3}\left[i\left(\omega_{1}-\omega_{0}-\Omega^{\prime}\right)+\Gamma_{13}\right]}\left\{\frac{1}{\gamma_{21}}\left[\frac{2 \Gamma_{12}}{\left(\omega_{0}-\omega_{2}\right)^{2}+\Gamma_{12}^{2}}\right]+\right.
$$

$$
\left.+\frac{1}{i\left(\omega_{1}-\omega_{2}-\Omega^{\prime}\right)+\Gamma_{23}}\left[\frac{1}{i\left(\omega_{1}-\omega_{0}-\Omega^{\prime}\right)+\Gamma_{13}}+\frac{1}{i\left(\omega_{0}-\omega_{2}\right)+\Gamma_{12}}\right]\right\} \text {. }
$$

Here, $E$ is the electric field amplitude, $\gamma$ and $\Gamma$ are corresponding damping constants. The inverse of the $\gamma$ and $\Gamma$ is the population and dephasing relaxation times, respectively. Note that $Q_{13}^{(3)}$ depends on both relaxation times, yielding a possibility of studying the relevant processes.

In the presence of very large inhomogeneous broadening, the analysis of (1) is simple. One can take the functions $g\left(\omega_{0}\right)$ and $g(\Omega)$ outside the integral and get the narrow almost homogeneous dispersion curve on a wide inhomogeneous pedestal $\left[{ }^{10,13}\right]$. In the case of not very large inhomogeneous broadening compared with the width of $(2)$, the integration must be performed assuming the functional form of the inhomogeneous distributions. According to the qualitative rule of $\left[{ }^{22}\right]$, one can readily see that in the case under study only electronic inhomogeneities, and not the vibrational ones, must be greatly reduced (there is a product of two resonant denominators with $\omega_{0}$ of opposite signs, whereas $\Omega^{\prime}$ are of the same signs).

These conclusions are confirmed by direct numerical calculations.

Fig. 1 The semilogarithmic dependence of the HWHM of $\left|P^{(3)}\left(\omega_{1}\right)\right|^{2}$ and $\left|P^{(1)}(\omega)\right|^{2}$ on the HWHM of inhomogeneous distributions for the input wave configurations indicated in the inserts. The values of the parameters used are $\gamma_{21}=2, \quad \Gamma_{12}=4$, $\Gamma_{13}=\Gamma_{23}=8$ and $\Delta \Omega / \Delta \omega=0,01$. The arbitrary units on both axes are equal.

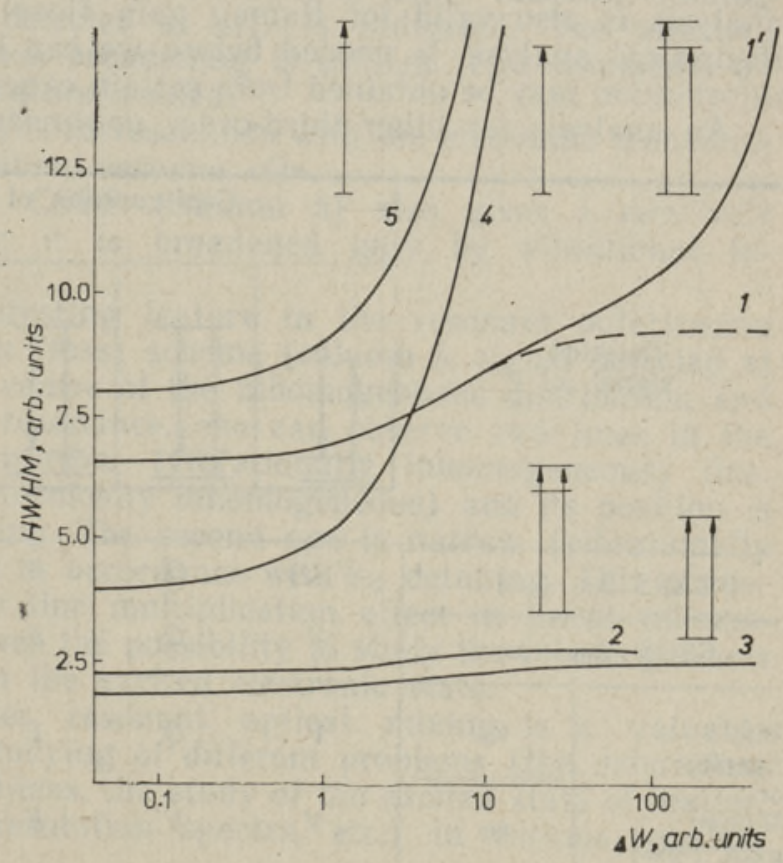


For the convience of integration both inhomogeneous distributions are approximated by Lorentzian curves with a peak at $\omega_{c}$ :

$$
g\left(\omega_{0}\right)=\Delta \omega / \pi\left[(\Delta \omega)^{2}+\left(\omega_{0}-\omega_{c}\right)^{2}\right] .
$$

Curve 1 in Fig. 1 is calculated, neglecting the vibrational inhomogeneities $(g(\Omega)=\delta$-function) and it shows that the electronic inhomogeneity is essentially reduced. Curve $1^{\prime}$ also includes the vibrational inhomogeneity with the ratio $\Delta \Omega / \Delta \omega=0,01$ and indicates that this kind of inhomogeneity is preserved. On the contrary, it will prevail at sufficiently large inhomogeneities. The vibrational inhomogeneity is surmountable if both laser frequencies are chosen close to a certain vibronic transition $\omega_{1} \approx$ $\approx \omega_{2} \approx \omega_{0}+\Omega^{\prime}$. If additionally, the substitions $\Gamma_{23} \rightarrow \gamma_{21}$ and $\Gamma_{12} \rightarrow \Gamma_{13}$ are made in (2), then it corresponds to the two level model of $\left[{ }^{13}\right]$ and shows a great reduction of both inhomogeneities (curve 2). Unfortunately this holds only for excited state vibrations (see below). Curve 3 is calculated on the assumptions that $\omega_{1} \approx \omega_{2} \approx \omega_{0}$ and $\Omega^{\prime}=0, \Gamma_{13} \rightarrow \Gamma_{12}$ and $\Gamma_{23} \rightarrow \gamma_{21}$. Then it gives again a narrow, almost inhomogeneity-free line, which shows that the approximation $g\left(\omega_{0}\right)=$ const, made in $\left[{ }^{13}\right]$ is correct (it causes only a small error in determining $\Gamma_{12}$ ).

For a comparison, in Fig. 1 the HWHM of the linear absorption lines are given using the same parameters (curves 4 and 5 ). This comparison clearly demonstrates the advantage of the third-order nonlinear spectroscopic methods for the study of fine homogenéous structures in the spectra of highly inhomogeneous media. If now the frequency $\omega_{2}$ of the probing wave is such that the difference $\omega_{2}-\omega_{0}$ is seanned through the vibrational levels of the ground state, the result is also only vibrationally inhomogeneous (see Table, column 2).

Taking into account what was stated above, there is a relatively simple method for obtaining a narrow-line spectrum from smooth inhomogeneous ones as well as supplying the necessary spectroscopic and dynamic information. The resonant character of processes guarantees a strong measurable signal. Concerning only the inhomogeneous properties, the analysis is also valid for Raman gain (loss). However, a very careful theoretical analysis is needed before we can know exactly what kind of information may be obtained from certain experiments.

An analysis for other third-order nonlinear processes was performed

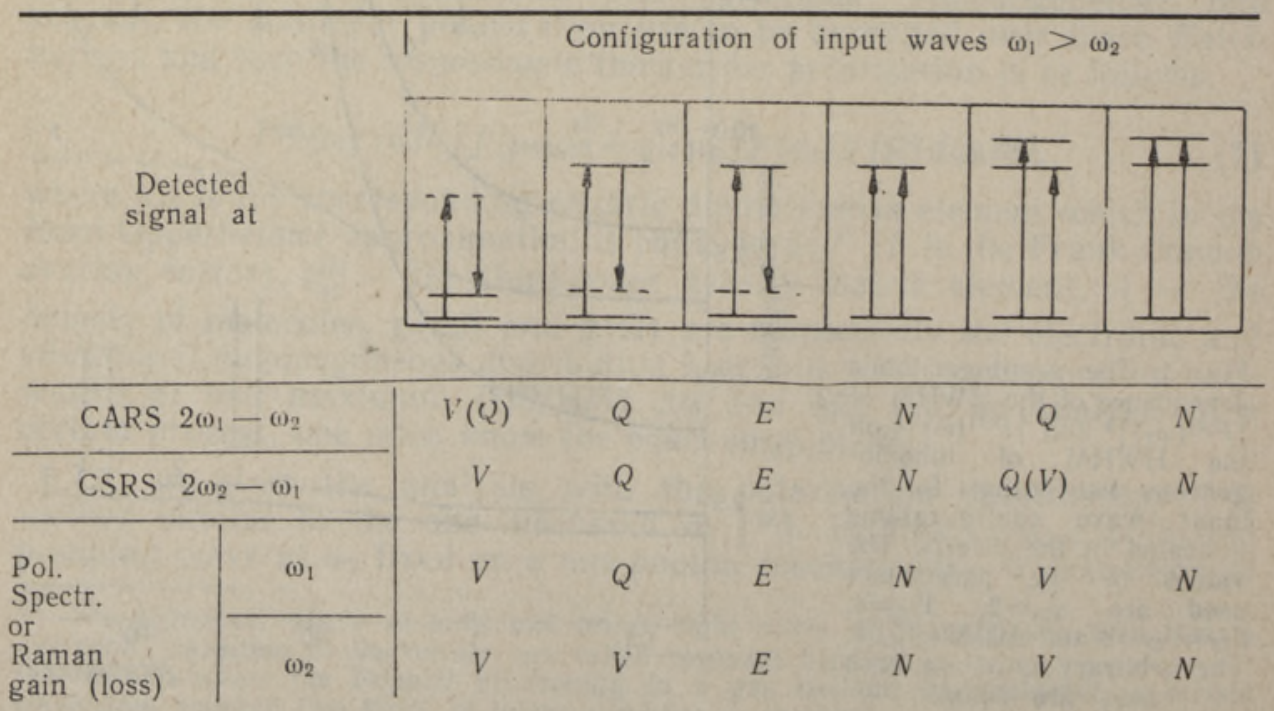


Fig. 2 The semilogarithmic dependence of the HWHM on the width of the electronic inhomogeneous distribution for the «Q» line. Damping constants for electronic and vibrational resonances are 4 and 8 units respectively.

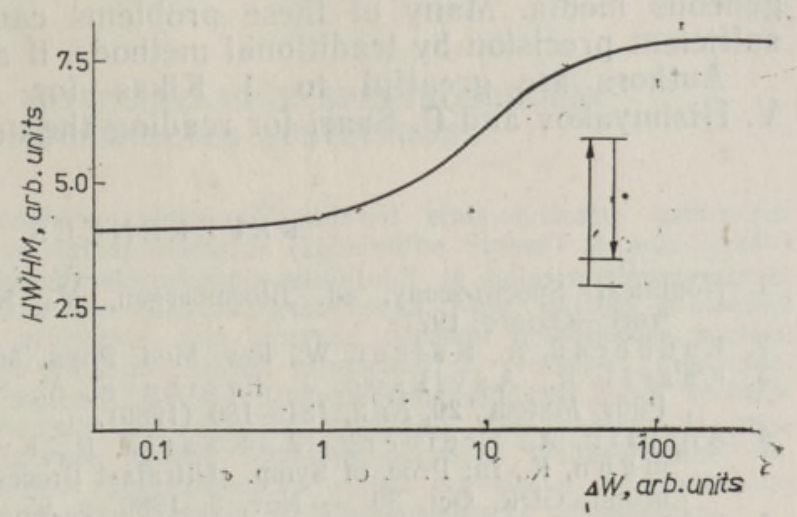

analogously, the results of which are summarized in the Table. In the energy diagrams solid and dotted lines denote respectively the real and virtual levels. The letters $\ll E »$ and $« V »$ label electronically and vibrationally inhomogeneous signals, respectively, «N» marks narrow-line signals (in this case both kinds of inhomogeneities are reduced).

As seen from the Table, all third-order nonlinear processes with $\omega_{1}-\omega_{2} \approx \Omega$ or $\Omega^{\prime}$ are at least vibrationally broadened. In many cases the spectra are also broadened by electronic inhomogeneities. But, and it is interesting to point out that if the formulas of nonlinear susceptibility contain a term with resonant vibrational denominator, it works so that the large electronic inhomogeneity in the corresponding spectral line is not manifest. In the Table these cases are marked with " $Q$. This property allows to observe relatively narrow lines corresponding to the vibrational resonance, independently of the width of the electronic inhomogeneous distribution.

As an example, in Fig. 2 the dependence of HWHM on the width of the electronic inhomogeneous distribution for a triply resonant mixing process (column 2, signal detected at $\omega_{1}$ ), is indicated. The effective suppression of inhomogeneous broadening is evident. Two comments on the CARS and CSRS spectra are needed:

1. If CARS signal $2 \omega_{1}-\omega_{2}$ is in resonance with the electronic transition $\omega_{0}$, then $\ll V »$ in the first column becomes $« Q »$.

2. If $\Omega=\Omega^{\prime}$, the resonant CSRS (column 5) also gives a relatively narrow-line « $V »$ spectrum: it is broadened only by vibrational inhomogeneities.

There is one more interesting feature in the resonant polarization spectroscopy or Raman gain (loss) scheme (column 5, signal detected at $\left.\omega_{1}\right)$. Detuning $\omega_{2}$ from the centre of the inhomogeneous distribution and scanning $\omega_{1}$ over vibronic resonance, one can observe two lines in the spectrum instead of one narrow (vibrationally inhomogeneous) line. One of them is broad (electronically inhomogeneous) and its position is not dependent on $\omega_{2}$ detuning. The second one is narrow (vibrationally inhomogeneous) and moves in accordance with $\omega_{2}$ detuning. This phenomenon is analogous to the line multiplication effect in linear inhomogeneous spectra $\left[{ }^{17}\right]$. It serves the possibility to study the inhomogeneous distribution of vibrations in the excited electronic state.

In conclusion, third-order resonant optical mixing is a valuable spectroscopic tool for the studying of different problems (the determination of ultrafast relaxation times, the study of the excited state of matter, the measurement of high-resolution spectra, etc.) in various inhomo- 
geneous media. Many of these problems cannot be resolved with a sufficient precision by traditional methods, if at all.

Authors are greatful to J. Kikas for valuable discussions and V. Hizhnyakov and P. Saari for reading the manuscript and remarks.

\section{REFERENCES}

1. Nonlinear Spectroscopy, ed. Bloembergen, N., North-Holland, Amsterdam-New York-Oxford, 1977.

2. L a uber a u, A., Ka i ser, W., Rev. Mod. Phys., 50, № 3, 607-665 (1978).

3. Ka a r li, R., Aaviksoo, J., Freiberg, A., S a ari, P., ENSV TA Toimet., Füüs. Matem., 29, № 2, 181-186 (1980).

4. Anijalg, A., Freiberg, A., Kaarli, R., Kukk, P., S a a ri, P., Timp $\mathrm{m}$ a n n, K., In: Proc. of Symp. «Ultrafast Processes in Spectroscopy», Reinhardsbrunn, GDR, Oct. 30 - Nov. 5, 1980, p. 95.

5. B r ewer, R. G., Phys. Today, № 5, 50-59 (1977).

6. С а м а р це в В. В., Ж. прикладной спектроскопии, 30, № 4, 581-611 (1979).

7. Reb a ne, K. K., Impurity Spectra of Solids, Plenum Press, New York-London, 1970.

8. Freiberg, A., Rebane, L. A., Phys. status solidi (b), 81, № 1, 359-369 (1977).

9. Y a j im a, T., Opt. Commun., 14, № 3, 378-382 (1975).

10. Y a j ima, T., S o uma, H., Phys. Rev., A17, № 1, 309-323 (1978).

11. Y a jim a, T., S o uma, H., I shida, Y., Phys. Rev., A17, № 1, 324-334 (1978),

12. S o u ma, H., T a ir a, Y., Y a j ima, T., In: Picosecond Phenomena, ed. Shank, C. V., Ippen, E. P., Shapiro, S. L., Springer-Verlag, Berlin-Heidelberg-New York, 1978 , p. $224-227$.

13. Song, J. J., Lee, J. H., Le ven so n, M. D., Phys. Rev., A17, № 4, 1439-1447 (1978).

14. Re ba ne, K., S a a ri, P., J. Luminescence, 16, № 3, 223-234 (1978).

15. Р еб ане Л. А., Фрей б ерг А. М., Изв. АН СССР, Сер. физ., 39, № 9, 19871992 (1975).

16. S z a b o, A., Phys. Rev. Lett., 25, № 14, 924-926 (1970).

17. А в а р м а а Р., Изв. АН ЭССР, Физ. Матем., 29, № 3, 238-247 (1974).

18. L e to khov, V. S., Ch e b o ta y e v, V. P., Nonlinear Laser Spectroscopy, Springer Verlag, Berlin, 1977.

19. Гороховский А. А., К а а ли Р. К., Ребане Л. А., Письма в ЖЭТФ, 20, вып. 7, 474-479 (1974).

20. Kharlamov, B. M., Personov, R. J., Bykovskaya, L. A., Opt. Commun., 12, № 1, 191-193 (1974).

21. Des ideri o, R. A., Hu d s o n, B. S., Chem. Phys. Lett., 61, № 4, 445-448 (1979).

22. Schlossberg, H. R., J avan, A., Phys. Rev., 150, № 1, 267-284 (1966); O u d a r, J.-L., Shen, Y. R., Phys. Rev., A22, № 3, 1141-1158 (1980).

23. Gorokhovski, A. A., Kikas, J., Opt. Commun., 21, № 2, 272-274 (1977),

24. George, S. M., A uw eter, H., Harris, C. B., J. Chem. Phys., 73, № 11, $5573-5583(1980)$.

25. Wi e ma n, C., Hänsch, T. W., Phys. Rev. Lett., 36, № 20, 1170-1173 (1976).

26. Andrews, J. R., Hochstrasser, R. M., Proc. Nat. Acad. Sci. USA, 77, № 6, $3110-3114$ (1980).

Academy of Sciences of the Estonian SSR, Institute of Physics

Received

May 21, 1981 


\section{STATSIONAARNE MITTELINEAARNE SPEKTROSKOOPIA MITTEHOMOGEENSETES SUSTEEMIDES}

Artiklis on analüüsitud ja mudelarvutustega põhjendatud statsionaarsete kolmandat järku mittelineaarsete optiliste resonantsprotsesside (koherentne Stokes'i ja anti-Stokes'i kombinatsioonhajumine, modulatsioonspektroskoopia amplituud- ja polarisatsioonivariant) sōltuvust elektron- ja võnkeseisundite mittehomogeensusest. Kõik uuritud protsessid võib jaotada kolme rühma: 1) protsessid, mis annavad peaaegu homogeense spektri: mõlemat tüüpi mittehomogeensuse mõju on oluliselt vähenenud; 2) protsessid, mis annavad kvaasihomogeense spektri: mōlemat tüüpi mittehomogeensused avalduvad spektris, kuid elektronseisundite mittehomogeensuse mõju on tunduvalt alla surutud; 3) protsessid, mis annavad mittehomogeense spektri. Mittehomogeensuse mahasurumine võimaldab efektiivsemalt kui lineaarses spektroskoopias uurida ergastatud elektronseisundeid ja ülikiireid relaksatsiooniprotsesse tugevalt mittehomogeensetes süsteemides.

П. КУКК, А. ФРЕИБЕРГ

\section{СТАЦИОНАРНАЯ НЕЛИНЕИНАЯ СПЕКТРОСКОПИЯ ТРЕТЬЕГО ПОРЯДКА В НЕОДНОРОДНЫХ СРЕДАХ}

В статье проанализировано и показано модельными расчетами, как электронная и колебательная неоднородности влияют на резонансные нелинейно-оптические процессы третьего порядка (когерентная активная спектроскопия комбинационного рассеяния, амплитудная и поляризационная модуляционная спектроскопия). Показано, что эти процессы можно разделить на три группы: 1) дающие однородные спектры - обе неоднородности значительно подавлены; 2) дающие «почти» однородные спектры обе неоднородности присутствуют в спектрах, но электронная не выявляется; 3) дающие неоднородные спектры. Подавление неоднородности позволяет более эффективно, чем в линейной спектроскопии, изучать возбужденные электронные состояния вещества и сверхбыстрые процессы релаксации в неоднородных средах. 